\title{
Valoración de la historia clínica electrónica por los profesionales de organizaciones sanitarias y sociales
}

\author{
Sergi Iglesia Reina ${ }^{a, b}$, Juan Uría González-Tovac,
} Ismael Cerdà Calafat ${ }^{d}$ y Francesc García Cuyàs ${ }^{e}$

DOI: 10.12961/aprl.2019.22.01.3

Recibido: 11 de junio de 2018

Aceptado: 15 de noviembre de 2018

\section{RESUMEN}

Objetivo: Evaluar diferencias en el grado de satisfacción de los profesionales que participan en la asistencia sanitaria y en la gestión de las organizaciones de salud en relación a la historia clínica electrónica (HCE) implantada en sus organizaciones.

Métodos: Se incluyen profesionales asistenciales, gestores y profesionales de Sistemas de Información que trabajan con la HCE de cualquier organización sanitaria o social en Cataluña. De 312 usuarios contactados, se obtuvo una tasa de participación del 60,25\%. Las variables explicativas fueron el sexo, la edad, nivel de estudios, tipo de organización y categoría profesional. Como variables dependientes se incluyeron: el grado de satisfacción global con el sistema actual, la valoración de la posibilidad de cambio de la aplicación de la HCE y la valoración de su uso para mejorar la atención sanitaria.

Resultados: Se observa un menor grado de satisfacción en los centros analizados respecto a atención primaria $(\mathrm{ORa}=0,11 ; \mathrm{IC} 95 \%=$ $(0,02-0,52))$, en los profesionales con Grado/Licenciatura $(\mathrm{ORa}=0,12 ; \mathrm{IC} 95 \%=(0,01-1,26))$ y de Máster/Doctorado $(\mathrm{ORa}=0,10 ; \mathrm{IC} 95 \%=(0,01$ 1,17) respecto a estudios secundarios, y en los profesionales considerados respecto a los de gerencia/dirección (Médica/o: ORa=0,18; IC $95 \%=(0,06-0,54)$ y Enfermera/o: ORa=0,36; IC 95\%=(0,13 - 1,03)).

Conclusiones: Las diferencias observadas entre los profesionales en su percepción del uso de la HCE deberían conllevar una mejora de estas herramientas para la asistencia de los pacientes.

PALABRAS CLAVE: Registros electrónicos de salud; continuidad de la atención al paciente; Tecnología de la Información.

\section{EVALUATION OF THE ELECTRONIC MEDICAL RECORD BY PROFESSIONALS IN HEALTH AND SOCIAL ORGANIZATIONS}

\begin{abstract}
Objective: To evaluate differences in the degree of satisfaction among professionals who participate in health care and in the management of health organizations in relation to the electronic medical record (EMR) used in their organizations.

Methods: We surveyed healthcare professionals, managers and information technology professionals who work with an EMR in any health or social organization in Catalonia. Among 312 contacted professionals, the participation rate was 60.25\%. Explanatory (independent) variables were sex, age, education, type of organization and professional category. Dependent variables included the degree of overall satisfaction with the current system, an assessment of possibly changing the EMR system s and an assessment of the use of an EMR to improve health care.

Results: There was a lower degree of satisfaction in primary care centers (sociosanitary, assisted residence and other work centers: ORa $=0.11,95 \% \mathrm{CI}=(0.02-0.52))$, among those with a professional or bachelor's degree $(\mathrm{ORa}=0.12,95 \% \mathrm{CI}=(0.01-1.26))$ and those with graduate studies $(\mathrm{ORa}=0.10,95 \% \mathrm{CI}=(0.01-1.17)$ as compared to respondents with secondary studies, and among healthcare professionals as compared to managers (physicians: $\mathrm{ORa}=0.18,95 \% \mathrm{CI}=(0.06-0.54)$ and nurses: $\mathrm{ORa}=0,36 ; 95 \% \mathrm{CI}=(0.13-1.03)$ ).

Conclusions: The differences observed among the professionals with respect to their perception of the use of the EMR should lead to improvements in the use of these tools for patient care.
\end{abstract}

KEYWORDS: electronic medical record; continuity of patient care; information technology

a. Universitat de Vic-Central de Catalunya, Vic, España.

b. MC Mutual, Barcelona, España.

c. Consorci Hospitalari de Vic, España.

d. CatSalut, Barcelona, España.

e. Grup de recerca Digital Care UVIC-UCC, Vic, España.
Autor de correspondencia:

Sergi Iglesia Reina

MC Mutual

C/ Provença, 321, séptima planta, 08037 Barcelona

sergi.iglesia@gmail.com 


\section{INTRODUCCIÓN}

El uso de las tecnologías de la información y comunicación (TIC, en adelante) ha dado lugar a la implantación de nuevos medios para la asistencia sanitaria. El crecimiento de aplicaciones web, la movilidad, la búsqueda automatizada, la inteligencia artificial, la realidad aumentada y la realidad virtual, han modificado la práctica asistencial ${ }^{1}$. Por ello, en los últimos años está desarrollándose un debate internacional sobre los beneficios de la aplicación de las TIC al ámbito sanitario. En este sentido, la Secretaría General de la Organización para la Cooperación y el Desarrollo Económico realizó un informe titulado "Mejora de la eficiencia del sector sanitario. El papel de las TIC”2. En él, se analizaba el uso de las TIC en la sanidad de seis países (Australia, Canadá, España, Estados Unidos, Países Bajos y Suecia) y concluyó que los principales beneficios de la implementación de las TIC en el sector sanitario son cuatro: 1) incremento de la calidad en la atención al paciente; 2) reducción de los costes operativos de los servicios médicos; 3) disminución de los costes administrativos y 4) posibilidad de llevar a cabo modelos de sanidad completamente nuevos.

Los profesionales sanitarios están inmersos en este proceso de cambios tecnológicos, que forma parte de su actividad profesional diaria. Las TIC, en este caso, tienen el propósito de reforzar, optimizar y facilitar la realización de las actuaciones sanitarias que, en definitiva, dan lugar a una atención de calidad. El proceso transformador de las TIC requiere de la continua formación y adaptación a sus puestos de trabajo ${ }^{3}$. Aunque las TIC pueden permitir a los profesionales organizarse su trabajo con mayor flexibilidad, también puede aumentar el riesgo de sufrir estrés laboral prolongado e insatisfacción laboral, lo que puede repercutir negativamente en su salud ${ }^{4,8}$.

Existen numerosos estudios sobre el perfil del paciente frente al crecimiento de las TIC en Sanidad, como es el caso del Observatorio Nacional de las Telecomunicaciones ${ }^{9}$. No obstante, hasta donde sabemos, no existen estudios que comparen la valoración de las TIC por parte de los profesionales asistenciales y de los gestores (como es el caso de la Historia Clínica Electrónica - HCE, en adelante-) en las organizaciones sanitarias y sociales a nivel nacional o autonómico.

El objetivo principal de este estudio es evaluar diferencias en el grado de satisfacción de los profesionales que participan en la asistencia sanitaria y en la gestión de las organizaciones de salud en relación a la HCE implantada en sus organizaciones.

\section{MÉTODOS}

\section{Diseño del estudio \\ Diseño transversal.}

\section{Lugar, tiempo y población}

La población de estudio fueron los profesionales asistenciales, gestores y profesionales de sistemas de información (profesionales vinculados a Informática, al servicio encargado del cuadro de mando corporativo o a aquellos profesionales que analizan los circuitos funcionales organizativos) que trabajan con la HCE de cualquier organización sanitaria o social. Se consideró como criterio de exclusión que el profesional encuestado que trabaja en una organización sanitaria o social catalana no estuviera en contacto con la HCE de su organización.

Para la selección de la muestra, desde la "Fundació TIC Salut i Social", se obtuvieron las direcciones de correo electrónico de todas las organizaciones sanitarias y sociales catalanas (fuesen públicas o privadas). Se invitó a participar al total de gerentes, directivos, mandos intermedios, profesionales asistenciales, profesionales vinculados a sistemas de información y, en general, a todos aquellos usuarios que trabajan con la HCE en las diferentes organizaciones sanitarias y sociales de Cataluña. Del sector público, respondieron un $92,89 \%$ de las organizaciones contactadas (de un total de 1252). Del sector privado, respondió un 0,78\% del total (91). Nos pusimos en contacto con las organizaciones que autorizaron proponer la encuesta a sus profesionales mediante la publicación en sus medios de comunicación internos al enlace del cuestionario o mediante direcciones de correo electrónico. De todos los centros que aprobaron el contacto con sus profesionales, 312 usuarios autorizaron que les enviásemos el cuestionario. El contacto se realizó entre los meses de enero y febrero de 2017.

\section{Instrumento de recogida de datos}

Se diseñó un cuestionario de 90 preguntas. El cuestionario permitió recoger datos sobre aspectos relacionados con la HCE. Para este estudio se seleccionaron un subconjunto de estas preguntas. El cuestionario recogió información en referencia a aspectos sociodemográficos, el uso de las TIC en relación a los datos de salud, la forma de acceder a la HCE, la valoración de la seguridad de la HCE de la organización donde trabajan, la valoración de la extracción de los datos necesarios para su trabajo, el contenido de la HCE y la visión del encuestado en cuanto al presente y al futuro de la HCE y de los datos de salud. El cuestionario completo está disponible bajo petición al autor de correspondencia.

Para contribuir a la evidencia de validez de contenido, se organizó un grupo de discusión con expertos. Este estaba constituido por el gerente de una organización sanitaria integrada, tres responsables de sistemas de información de diferentes organizaciones sanitarias, dos proveedores tecnológicos, dos médicos y dos enfermeros. Se obtuvo evidencia de validez de constructo mediante el pase del cuestionario a veinte personas (profesionales vinculados a la Informática, medicina y enfermería) de tres organizaciones sanitarias de Cataluña, realizando test-retest y observando consistencia en las respuestas antes y después. El tiempo promedio para contestar la totalidad del cuestionario fue de 30 minutos.

El cuestionario estaba disponible en un enlace en línea (online). Se diseñó y facilitó a los participantes mediante la herramienta LimeSurvey, que estaba hospedada en los servidores de la Universitat de Vic. Se trató de una encuesta anónima que no facilitaba credenciales de acceso a los usuarios. El control para evitar repeticiones de la encuesta por un mismo usuario era la detección de la dirección de IP utilizada por el usuario. Si el sistema detectaba la misma IP, no permitía realizar nuevamente el cuestionario.

\section{Variables de estudio}

Se consideraron como variables explicativas el sexo, la edad (20-39 años, 40-59 y $\geq 60$ años), nivel de estudios (estudios secundarios, Grado/Licenciatura y máster/doctorado), tipo de organización donde trabajan (Organización Sanitaria Integrada, Agudos/Especializada, Primaria y Otros (Sociosanitario, residencia asistida y otros centros de trabajo)) y categoría profesional (Gerencia/Dirección, Médica/co, Enfermera/o, Profesional Sistemas 
de Información y Otros). En el grupo de gerencia y dirección (así como mandos intermedios) participan todos aquellos profesionales que se dedican exclusivamente a tareas de gestión en sus organizaciones En el grupo de profesionales de sistemas de información se pueden encontrar informáticos, analistas de datos, estadísticos o ingenieros. En el grupo "otros", se incluyen trabajadores sociales, terapeutas ocupacionales y psicólogos. Como variables dependientes se incluyeron:

- El grado de satisfacción global con la aplicación actual de HCE de la organización por parte del profesional. Esta variable tomaba valores de 1 (nada satisfecho) a 5 (muy satisfecho). Se agrupó en grado de satisfacción "alta" (puntuación 4 y 5) y satisfacción "baja” (puntuación 1, 2 y 3). Se consideró medir la frecuencia de satisfacción alta.

- Valoración de la posibilidad de cambio de la aplicación la HCE de su organización. La pregunta realizada fue ¿Cambiaría el sistema actual de HCE de su organización por otro? La pregunta tomó valores "sí", "no" y "no estoy seguro". Se agruparon las opciones no y no estoy seguro, considerando medir la frecuencia de sí.

- Valoración del uso de la HCE del paciente para mejorar la atención del momento. La pregunta realizada fue ¿Cree que el acceso a la HCE del paciente que el profesional sanitario realizará durante la visita mejorará la atención del momento? La pregunta tomó valores en una escala de likert con opciones "siempre", "casi siempre", "a menudo", "pocas veces" y "nunca/casi nunca". Se agruparon las opciones "siempre", "casi siempre” y "a menudo" en un grupo considerado como grado de valoración "alta”, y "pocas veces" y "nunca/casi nunca" en otro grupo considerado como valoración "baja". Se midió la frecuencia de valoración "alta".

\section{Análisis estadístico}

Se calculó la proporción de profesionales que cambiarían el sistema actual de HCE de su organización por otro, la proporción con valoración alta para el uso de la HCE del paciente para mejorar la atención del momento, y la proporción que reportaron satisfacción alta con el sistema actual de HCE de la organización. Estas proporciones se estimaron para las variables explicativas. Finalmente, se realizaron dos modelos de regresión logística para la proporción de profesionales que cambiarían el sistema actual de HCE y la proporción que reportaron satisfacción alta con el sistema actual de HCE, respectivamente. Se obtuvieron las odds ratio e intervalos de confianza a los $95 \%$ ajustadas por todas las variables explicativas.

No se realizó un modelo estadístico para la proporción que reportaron valoración alta para el uso de la HCE del paciente para mejorar la atención del momento debido a que las proporciones observadas eran muy elevadas en todos los grupos comparados. Para realizar los análisis se utilizó el programa estadístico R10.

\section{Aspectos éticos}

Se envió el consentimiento informado por correo electrónico a los participantes. Una vez aceptaron a participar en el estudio se les facilitaba el enlace al cuestionario. La recogida de información preservó el anonimato del entrevistado.

\section{RESULTADOS}

\section{Características de los participantes}

De 312 contactados, 285 profesionales aceptaron responder la encuesta. No obstante, 188 respondieron el cuestionario en su totalidad, obteniendo una tasa de participación del 60,25\%. Un 67,20\% pertenecían a la provincia de Barcelona, un 28,49\% a Girona y, en menor medida, están las provincias de Lleida y Tarragona con únicamente un 2,15\% cada una.

La distribución por sexo (tabla 1) es ligeramente superior en mujeres $(55,9 \%)$, con el rango de edad mayoritario situado entre 40 y 59 años (67,0\%). Prácticamente no hay participantes con estudios secundarios (3,2\%), situándose el grupo mayor en aquellos con estudios universitarios (53,2\%). Respecto al centro de trabajo, el grupo mayor (41,5\%) trabaja en centros de Agudos o de Atención Especializada. Para el perfil profesional destaca que el 29,8\% de los encuestados es del colectivo de Enfermería, seguidos con un porcentaje similar (entre el $18,1 \%$ y $19,7 \%$ ) por Sistemas de Información, Gerencia o Dirección de organizaciones sanitarias y colectivo médico.

TABLA 1

Características de los participantes (n=188). Cataluña, 2017.

Variables

n

(\%)

Sexo

\begin{tabular}{lll}
\hline Mujer & 105 & $(55,9)$ \\
\hline Hombre & 83 & $(44,1)$ \\
\hline
\end{tabular}

Edad (años)

\begin{tabular}{lll}
\hline $20-39$ & 46 & $(24,5)$ \\
\hline $40-59$ & 126 & $(67,0)$ \\
\hline$\geq 60$ & 16 & $(8,5)$
\end{tabular}

Nivel de estudios

\begin{tabular}{lll}
\hline $20-39$ & 46 & $(24,5)$ \\
\hline $40-59$ & 126 & $(67,0)$ \\
\hline$\geq 60$ & 16 & $(8,5)$ \\
\hline
\end{tabular}

Nivel de estudios

\begin{tabular}{lll}
\hline Estudios secundarios & 6 & $(3,2)$ \\
\hline Grado/Licenciatura & 100 & $(53,5)$ \\
\hline Estudio Máster/Doctorado & 82 & $(43,6)$ \\
\hline
\end{tabular}

Centro de trabajo

\begin{tabular}{lll}
\hline Organización Sanitaria Integrada & 58 & $(30,9)$ \\
\hline Agudos/Especializada & 78 & $(41,5)$ \\
\hline Primaria & 14 & $(7,4)$
\end{tabular}

Perfil Profesional

\begin{tabular}{lll}
\hline Gerencia/Dirección & 35 & $(18,6)$ \\
\hline Médica/co & 37 & $(19,7)$ \\
\hline Enfermera/o & 56 & $(29,8)$ \\
\hline $\begin{array}{l}\text { Profesional Sistemas } \\
\text { de Información/Informática }\end{array}$ & 34 & $(18,1)$ \\
\hline Otros & 26 & $(13,8)$ \\
\hline Total & $\mathbf{1 8 8}$ & $(100,0)$ \\
\hline
\end{tabular}

1. Sociosanitario, residencia asistida y otros centros de trabajo. 
En la valoración del grado de satisfacción con el sistema actual de HCE de su organización, se observa que, únicamente, el 36,7\% de los usuarios presenta un grado alto de satisfacción con la HCE. En relación a las variables analizadas, se observan diferencias estadísticamente significativas en el nivel de estudios $(p=0,067)$, centro de trabajo $(p=0,002)$ y perfil profesional $(p=0,022)$. En el caso del nivel de estudios el $83,3 \%$ de los profesionales con estudios secundarios presentan un grado de satisfacción alto, frente a aproximadamente un 35\% en estudios de grado/licenciatura y de máster/doctorado. En relación a la satisfacción de la HCE por centro de trabajo se observa que el 50\% de los profesionales de Atención Primaria presenta un grado alto de satisfacción, así como sucede con el 48,28\% de los profesionales que pertenecen a organizaciones sanitarias integradas. Sin embargo, en centros sociosanitarios, residencia asistida y otros centros de trabajo es únicamente de un 13,2\%. En cuanto a la valoración de la HCE por categoría profesional, se observa que la frecuencia más elevada de satisfacción se da en los profesionales de Gerencia o en el Comité Directivo, y la menor en médicos (57,1\% frente a un 21,6\%). Cuando consideramos todas las variables conjuntamente en el modelo multivariable, no se observan cambios significativos en los re-

\section{TABLA 2}

Número y proporción de profesionales que consideran que con una alta frecuencia que el acceso a la HCE del paciente mejorará la atención del paciente, que cambiarían el sistema actual de HCE de su organización y que presentan un alto grado de satisfacción global con el sistema actual de HCE de su organización. Cataluña, 2017.

¿Cree que el acceso a la $\mathrm{HCE}$ del paciente que el profesional sanitario realizará durante la visita mejorará la atención del momento?
¿Cambiaría el sistema actual de HCE de su organización por otro?
Grado de Satisfacción global con el sistema actual de HCE de su organización

\begin{tabular}{|c|c|c|c|c|c|c|c|c|c|}
\hline Variables & número 2 & $(\%)$ & $\mathrm{p}$ & número $^{3}$ & $(\%)$ & $\mathrm{p}$ & número ${ }^{4}$ & $(\%)$ & $\mathrm{p}$ \\
\hline \multicolumn{10}{|l|}{ Sexo } \\
\hline Mujer & 99 & $(94,3)$ & 0,136 & 56 & $(53,3)$ & 1,000 & 34 & $(32,3)$ & 0,174 \\
\hline Hombre & 82 & $(98,8)$ & & 45 & $(54,2)$ & & 35 & $(42,2)$ & \\
\hline \multicolumn{10}{|l|}{$\underline{\text { Edad (años) }}$} \\
\hline $20-39$ & 44 & $(95,7)$ & 1,000 & 32 & $(69,6)$ & 0,040 & 16 & $(34,8)$ & 0,970 \\
\hline $40-59$ & 121 & $(96,0)$ & & 61 & $(48,4)$ & & 47 & $(37,3)$ & \\
\hline$\geq 60$ & 16 & $(100,0)$ & & 8 & $(50,0)$ & & 6 & $(37,5)$ & \\
\hline \multicolumn{10}{|l|}{ Nivel de estudios } \\
\hline Estudios secundarios & 6 & $(100,0)$ & 1,000 & 1 & $(16,7)$ & 0,211 & 5 & $(83,3)$ & 0,067 \\
\hline Grado/Licenciatura & 96 & $(96,0)$ & & 55 & $(55,0)$ & & 35 & $(35,0)$ & \\
\hline Estudios Máster/Doctorado & 79 & $(96,3)$ & & 45 & $(54,9)$ & & 29 & $(35,4)$ & \\
\hline \multicolumn{10}{|l|}{ Centro de Trabajo } \\
\hline Organización Sanitaria Integrada & 58 & $(100,0)$ & 0,123 & 29 & $(50,0)$ & $<0,001$ & 28 & $(48,3)$ & 0,002 \\
\hline Agudos/Especializada & 73 & $(93,6)$ & & 39 & $(51,3)$ & & 29 & $(37,2)$ & \\
\hline Primaria & 13 & $(92,9)$ & & 2 & $(14,3)$ & & 7 & $(50,0)$ & \\
\hline Otros & 37 & $(97,4)$ & & 31 & $(81,6)$ & & 5 & $(13,2)$ & \\
\hline \multicolumn{10}{|l|}{ Perfil Profesional } \\
\hline Gerencia/Dirección & 35 & $(100,0)$ & 0,122 & 16 & $(45,7)$ & 0,703 & 20 & $(57,1)$ & 0,022 \\
\hline Médica/co & 34 & $(91,9)$ & & 20 & $(54,1)$ & & 8 & $(21,6)$ & \\
\hline Enfermera/o & 52 & $(92,9)$ & & 31 & $(55,4)$ & & 18 & $(32,1)$ & \\
\hline $\begin{array}{l}\text { Profesional Sistemas de Información } \\
\text { /Informática }\end{array}$ & 34 & $(100,0)$ & & 17 & $(50,0)$ & & 15 & $(44,1)$ & \\
\hline Otros & 26 & $(100,0)$ & & 17 & $(65,4)$ & & 8 & $(30,8)$ & \\
\hline Total & 181 & $(96,3)$ & & 101 & $(53,7)$ & & 69 & $(36,7)$ & \\
\hline
\end{tabular}

1. Sociosanitario, residencia asistida y otros centros de trabajo.

2. Número de respuestas con valoración alta (siempre/casi siempre/a menudo).

3. Número de respuestas Sí. $\quad 4$. Número de respuestas con satisfacción alta (puntuación 4 y 5 ).

p. Obtenido mediante la prueba exacta de Fischer. 
sultados descritos (Tabla 3); es decir, se mantienen las diferencias observadas en el grado de satisfacción global con el sistema actual de HCE de la organización según centro de trabajo y perfil profesional ajustando por el resto de variables. Específicamente, se observa un menor grado de satisfacción en los centros analizados respecto a atención primaria (Organización Sanitaria: ORa $=0,58$; IC 95\% $=(0,15-2,26)$, Agudos/Especializada: ORa $=0,54$; IC 95\%=(0,15 - 1,96) y Otros (Sociosanitario, residencia asistida y otros centros de trabajo): $\mathrm{ORa}=0,11$; IC $95 \%=(0,02-0,52))$, en los de Grado/Licenciatura $(\mathrm{ORa}=0,12$; IC $95 \%=(0,01-1,26))$ y de máster/doctorado $(O R a=0,10 ;$ IC $95 \%=(0,01-1,17)$ respecto a estudios secundarios, y en los profesionales considerados respecto a los de gerencia/dirección (Médica/o: ORa=0,18; IC 95\%=(0,06-0,54) y Enfermera/o; ORa=0,36; IC 95\%=(0,13 - 1,03)).
Al analizar los resultados en relación a la opinión de los encuestados respecto a cambiar el sistema actual de HCE de su organización por otro, observamos que aproximadamente la mitad $(53,7 \%)$ cambiaría dicho sistema. Respecto a la variables explicativas consideradas, se obtienen diferencias estadísticamente significativas en la edad $(p=0,040)$ y en centro de trabajo ( $p<0,001$ ). En relación a la edad, se observa que el 69,6\% de los usuarios con una edad que oscila entre los 20 y 39 años opinan que cambiarían su herramienta de trabajo para la asistencia, frente a aproximadamente un $50 \%$ en los mayores de 39 años. Según tipo de organización a la que pertenecen los usuarios encuestados, se observa que el $81,6 \%$ de los profesionales que trabajan en organizaciones como Sociosanitarios, Salud Mental, Residencias o Mutuas Laborales, cambiarían la HCE, seguidos de aproximadamente un $50,0 \%$ en los profesionales que pertenecen a orga-

TABLA 3

Asociación (Odds Ratio e intervslo de confianza al 95\%) entre cambiar el sistema actual de HCE de la organización

y el alto grado de satisfacción global con dicho sistema. Cataluña, 2017.

¿Cambiaría el sistema actual de HCE de su organización por otro?
Grado de Satisfacción global con el sistema actual de HCE de su organización ${ }^{3}$

\begin{tabular}{|c|c|c|c|c|c|c|c|c|}
\hline Variables & ORa & $(\mathrm{IC} 95 \%)$ & & $\mathrm{p}$ & ORa & $(\mathrm{IC} 95 \%)$ & & $\mathrm{p}$ \\
\hline \multicolumn{9}{|l|}{ Sexo } \\
\hline Mujer & 0,95 & $(0,46-1,94)$ & & 0,878 & 0,76 & $(0,37-1,59)$ & & 0,474 \\
\hline Hombre & 1 & & & & 1 & & & \\
\hline \multicolumn{9}{|l|}{ Edad (años) } \\
\hline $20-39$ & 2,37 & $(0,66-8,58)$ & & 0,096 & 1,73 & $(0,43-6,87)$ & & 0,655 \\
\hline $40-59$ & 1,01 & $(0,33-3,07)$ & & & 1,24 & $(0,37-4,22)$ & & \\
\hline$\geq 60$ & 1 & & & & 1 & & & \\
\hline \multicolumn{9}{|l|}{ Nivel de estudios } \\
\hline Estudios secundarios & 1 & & & 0,324 & 1 & & & 0,113 \\
\hline$\underline{\text { Grado/Licenciatura }}$ & 5,30 & $(0,47-60,22)$ & & & 0,12 & $(0,01-1,26)$ & & \\
\hline Estudios Máster/Doctorado & 4,66 & $(0,40-54,27)$ & & & 0,10 & $(0,01-1,17)$ & & \\
\hline \multicolumn{9}{|l|}{ Centro de Trabajo } \\
\hline Organización Sanitaria Integrada & 7,41 & $(1,37-39,98)$ & ** & $<0,001$ & 0,58 & $(0,15-2,26)$ & & 0,004 \\
\hline Agudos/Especializada & 5,72 & $(1,13-28,93)$ & $* *$ & & 0,54 & $(0,15-1,96)$ & & \\
\hline Primaria & 1 & & & & 1 & & & \\
\hline Otros & 30,53 & $(4,98-187,27)$ & ** & 0,11 & $(0,02$ & & $* *$ & \\
\hline \multicolumn{9}{|l|}{ Perfil Profesional } \\
\hline Gerencia/Dirección & 1 & & & 0,876 & 1 & & & 0,036 \\
\hline Médica/co & 1,35 & $(0,48-3,82)$ & & & 0,18 & $(0,06-0,54)$ & $* *$ & \\
\hline Enfermera/o & 1,04 & $(0,37-2,92)$ & & & 0,36 & $(0,13-1,03)$ & * & \\
\hline $\begin{array}{l}\text { Profesional Sistemas de Información } \\
\text { /Informática }\end{array}$ & 0,74 & $(0,24-2,221)$ & & & 0,53 & $(0,17-1,60)$ & & \\
\hline Otros & 1,13 & $(0,33-3,88)$ & & & 0,42 & $(0,12-1,48)$ & & \\
\hline
\end{tabular}

1. Sociosanitario, residencia asistida y otros centros de trabajo. 2. Número de respuestas con valoración alta (siempre/casi siempre/a menudo). 3. Número de respuestas Sí. 4. Número de respuestas con satisfacción alta (puntuación 4y 5). ORa. Odds ratio ajustado por todas las variables explicativas de la tabla. IC95\%. Intervalo de confianza 95\%. p. Obtenido mediante la prueba de razón de verosimilitudes. ${ }^{*} \mathrm{p}<0,10 ;{ }^{*} \mathrm{p}<0,05$. 
nizaciones de Atención Especializada, y únicamente un 14,3\% en los centros de atención primaria. También para este caso, no se observan cambios significativos en los resultados descritos al considerar todas las variables conjuntamente en el modelo multivariable (Tabla 3). Específicamente, los profesionales de menor edad cambiarían con mayor frecuencia el sistema actual de HCE de su organización (20-39 años respecto a 60 o más: $O R a=2,37$; IC $95 \%=(0,66-8,58))$. Por otro lado, los profesionales de los centros de trabajo analizados cambiarán con mayor frecuencia la HCE respecto a los profesionales de atención primaria (Organización Sanitaria: ORa=7,41; IC 95\%=(1,37-39,98), Agudos/Especializada: ORa=5,72; IC 95\%=(1,13 - 28,93) y Otros (Sociosanitario, residencia asistida y otros centros de trabajo): ORa=30,53; IC 95\% $=(4,98-187,27))$.

Por último, un 96,3\% de los profesionales (ver tabla 2) consideran que el acceso a la HCE del paciente realizada durante la consulta mejorará la atención del momento. Esta elevada proporción se mantiene en todas las variables.

\section{DISCUSIÓN}

Los resultados muestran un menor grado de satisfacción con la HCE en los centros analizados (Organización sanitaria integrada, agudos/especializada, sociosanitario, residencia asistida y otros centros de trabajo) respecto a atención primaria, en los estudios universitarios y de máster/doctorado respecto a estudios secundarios, y en los profesionales considerados respecto a los de gerencia/dirección. Por otro lado, los profesionales de menor edad respecto a los de más edad, y los de los centros de trabajo analizados respecto a atención primaria, son más partidarios de cambiar la HCE. Es escasa la evidencia científica en relación a las evaluaciones de los sistemas de Historia Clínica Electrónica. Existe un estudio previo sobre la Historia Clínica Compartida de Cataluña ${ }^{11}$, pero no así de la Historia Clínica Electrónica utilizada en las diferentes organizaciones sanitarias y sociales catalanas. No obstante, podemos destacar que en una revisión sistemática de los sistemas de Historia Clínica Electrónica de Holanda se demuestra que el uso de éstos aumenta la satisfacción de usuarios y pacientes, aunque el efecto en los resultados clínicos no fueron concluyentes $^{12}$. Los resultados de la baja valoración del grado de satisfacción por parte de los usuarios en relación a la HCE, contrasta con estudios previos (Galimany-Masclans ${ }^{13}$, Olvera-Lobo ${ }^{14}$ ), donde la gran mayoría de los profesionales encuestados valoran con un nivel intermedio-alto el uso de la HCE. Este dato, junto al hecho que la mayoría de los usuarios encuestados cambiaría la aplicación actual de Historia Clínica Electrónica de su organización por otro (un 53,7\% del total), son datos que los fabricantes y desarrolladores de productos de Historia Clínica Electrónica deben tener en cuenta para el futuro.

Una posible explicación a las diferencias encontradas puede ser debida a que la introducción de la HCE en el sector salud genera una serie de cambios en la forma de $\operatorname{trabajar}^{13}$ que podría afectar de forma diferente a los profesionales implicados. Asimismo, otra posible explicación a las diferencias encontradas es que el nivel de satisfacción con la HCE está íntimamente relacionado con nivel de estrés laboral en cuanto a la intención de reducir las horas de trabajo por parte de los profesionales asistenciales, tal como evidencia un estudio realizado con los médicos de Estados Unidos $^{15}$, así como con las ineficiencias detectadas por los profesionales asistenciales en relación a los circuitos de trabajo una vez implantada la HCE ${ }^{16,18}$.

Como limitaciones del estudio se puede considerar que se han seleccionado únicamente los profesionales sanitarios de la Comunidad Autónoma catalana, debido a que sólo se disponían de los datos de contacto de las entidades catalanas por parte de la Fundació TIC Salut i Social. Otra posible limitación del estudio es la pérdida de individuos contactados para la realización de la encuesta. Al tratarse de una encuesta anónima, los investigadores no hemos podido realizar un seguimiento exhaustivo para que todos los usuarios contactados y que autorizaron el envío de la encuesta respondiesen las preguntas de esta última.

Por otro lado, la estrategia de selección de la muestra ha usado un muestreo no probabilístico ${ }^{19}$. Utilizar un muestro no probabilístico en el cual han participado voluntarios hace que los resultados obtenidos de la frecuencia de satisfacción, el uso de la HCE del paciente para mejorar la atención del momento, y la posibilidad de cambiar aspectos del sistema actual de la HCE de su organización no tengan por qué ser representativos de la población de profesionales sanitarios. También puede originar sesgos la imposibilidad de contar con profesionales con otros modelos de HCE en Cataluña diferentes a los contemplados en este estudio. A pesar de estas limitaciones, consideramos que los resultados obtenidos en los cuales se observan diferencias entre los diferentes profesionales en su percepción del uso de la HCE, son útiles para plantear acciones de mejora en las organizaciones sanitarias que pueden afectar a una parte de los usuarios ${ }^{20,21}$.

En este sentido, los resultados obtenidos en este estudio en relación a la valoración de la herramienta de la HCE por parte de los usuarios y la posibilidad de cambio de estas herramientas, deben permitir a la Administración Pública, a los gestores de entidades sanitarias y sociales (independientemente, del tipo de financiación) y a los proveedores de aplicaciones de HCE, analizar la voluntad de los profesionales sanitarios de mejorar las herramientas de trabajo para la asistencia y el cuidado de los pacientes. 


\section{REFERENCIAS}

1. Aranda F, González S, Roldán R. Influencia de las nuevas tecnologías en la promoción de la salud. 3er Congreso Internacional de Comunicación en Salud; 19-20 octubre 2017. Madrid: Universidad Carlos III [citado 2 Nov 2018]. Disponible: http: //e-archivo.uc3m.es/bitstream/handle/10016/25532/influencia_aranda_3ICHC_2017.pdf?sequence $=1$.

2. Improving Health Sector Efficiency: The Role of Information and Communication Technologies [edición electrónica]. 2010 [citado 2 Nov 2018]. Disponible en https://www.oecd.org/health/health-systems/improvinghealthsectorefficiency.htm. 3. Arandojo MI. Nuevas Tecnologías y nuevos retos para el profesional de enfer mería. Index Enferm Internet. Index Enferm. 2016; 25, 1-2:38-41.

4. Ninaus K, Diehl S, Terlutter R, Chan K, Huang A. Benefits and stressorsPerceived effects of ICT use on employee health and work stress: An exploratory study from Austria and Hong Kong, Sweden. Int J Qual Stud Health Well-being. 2015; $12: 28838$.

5. Escriba-Aguir V, Artazcoz L, Pérez-Hoyos S. Efecto del ambiente psicosocial y de la satisfacción laboral en el síndrome de burnout en médicos especialistas. Gaceta Sanitaria. 2008; 22:300-308.

6. Day A, Paquet S, Scott N, Hambley L. J Occup Health Psychol. 2012; 17:473-91 7. Smith M, Conway F, Karsh B. Occupational stress in human computer interaction. Ind Health. 1999; 37:157-73.

8. Stadin M, Nordin M, Broström A, Magnusson L, Westerlund H, Fransson E. Information and communication technology demands at work: the association with job strain, effort-reward imbalance and self-rated health in different socioeconomic strata. Int Arch Occup Environ Health. 2016; 89(7):1049-58.

9. Vázquez R, Martínez M. Estudio sobre opiniones y expectativas de los ciudadanos sobre el uso y la aplicación de las TI en el ámbito sanitario. [Edición electrónica]. 2015 [citado 2 Nov 2018]. Disponible en: https://www.ontsi.red.es/ ontsi/sites/ontsi/files/los_ciudadanos_ante_la_e-sanidad.pdf.

10. R Core Team (2016). R: A language and environment for statistical computing. R Foundation for Statistical Computing, Vienna, Austria. Disonible en https://www.R-project.org/.

11. Marimon S, Rovira M, Acedo M, Nozal MA, Guanyabens J. Shared electronic health record in Catalonia, Spain. Med Clin. 2010; 134 (Suppl 1): 45-48.

12. Van der Bij S, Khan N, Ten Veen P, de Bakker DH3, Verheij RA. Improving the quality of HER recording in primary care: A data quality feedback tool. J Am Med Inform Assoc. 2017; 24:81-87.

13. Galimany-Masclans J, Garrido-Aguilar E, Girbau-García MR, Lluch-Canut T, Fabrellas-Padrés N. New technologies and nursing: use and perception of primary healthcare nurses about electronic health record in Catalonia, Spain. Telemed J E Health. 2011; 17: 635-9.

14. Olvera-Lobo MD, Benítez-de-Vandrell B. Aproximación a las actitudes y percepciones de los usuarios ante las tecnologías de la información. El profesional de la información. 2008; 17: 199-204.

15. Sinsky CA, Dyrbye LN, West CP, Satele D, Tutty M, Shanafelt TD. Professional Satisfaction and the Career Plans of US Physicians. Mayo Clin Proc. 2017; 92:1625-1635.

16. Friedberg MW, Chen PG, Van Busum KR, Aunon F, Pham C, Caloyeras J et al. Factors Affecting Physician Professional Satisfaction and Their Implications for Patient Care, Health Systems, and Health Policy. Rand Health Q. 2014; 3:1 17. Rao SK, Kimball AB, Lehrhoff SR, Hidrue MK, Colton DG, Ferris TG, et al. The impact of administrative burden on academic physicians: results of a hospital wide physician survey. Acad Med. 2017; 92:237-243.

18. Shanafelt TD, Dyrbye LN, Sinsky C, Hasan O, Satele D, Sloan J, et al. Relationship between clerical burden and characteristics of the electronic environment with physician burnout and professional satisfaction. Mayo Clin Proc. 2016; 91:836-848.

19.Non-probability Sampling. Statistics Canada. Disponible en: https://www.statcan. gc.ca/edu/ power-pouvoir/ ch13/ nonprob/ 5214898-eng.htm (citado 2 Nov 2018).

20. Rothman KJ. Six persistent research misconceptions. J Gen Intern Med. 2014; 29(7): 1060-7.

21. Rothman KJ, Gallacher JE, Hatch EE. Why representativeness should be avoided. Int J Epidemiol. 2013; 42: 1012-4.
METROLOGÍA EN TIEMPO REAL PARA LA PREVENCIÓN DEL RIESGO QUÍMICO 12 de febrero de 2019, París (Francia)

\section{Información:}

E-mail: metro-tempsreel2019@inrs.fr

Web: https://www.inrs-metro-tempsreel2019.fr/

\section{CONGRESO NACIONAL DE MEDICINA Y ENFERMERÍA DEL TRABAJO CNMET 19 \\ 14-16 de febrero de 2019, Madrid}

Información:

Paseo del General Martínez Campos, 9, 2, 28010 Madrid, España.

Tel.: (34) 913.836 .000

E-mail: cnmet2019@pacifico-meetings.com

Web: www.cnmet2019.com/index.php

SALUD Y BIENESTAR EN EL TRABAJO

5-6 de marzo de 2019, Birmingham (Reino Unido)

Información:

Web: http://www.healthwellbeingwork.co.uk/

CONCEPTO SIMPLE DE MEDIDA

DE SUSTANCIAS PELIGROSAS:

TALLER PARA USUARIOS

6-7 de marzo de 2019, Dortmund (Alemania)

\section{Información:}

Elke Büdeker, Bundesanstalt für Arbeitsschutz und Arbeitsmedizin (BAuA)Friedrich-Henkel-Weg 1-25, 44149, Dortmund, Alemania. Tel.: 0231 9071-2322. Fax: 0231 9071-2611

E-mail: sek4.6@baua.bund.de

Web: https://www.baua.de/DE/Angebote/Veranstaltungen /Termine/2019/03.06-EMKG.html

\section{TRABAJADORES EN AMBIENTE FRÍO}

19-21 de marzo de 2019, Tromsø (Noruega)

\section{Información:}

Linda Vänskä, NIVA, Arinatie 3A, FI-00370 Helsinki, Finlandia.

Tel.: +358405463277

E-mail: linda.vanska@niva.org

Web: https://niva.org/course/workers-in-the-cold/ 